\title{
El CEDEPCI. Una propuesta para incentivar el pensamiento científico en la escuela
}

THE CEDEPCI. A PROPOSAL TO ENCOURAGE SCIENTIFIC THINKING IN SCHOOL

O CEDEPCI. UMA PROPOSTA PARA INCENTIVAR O PENSAMENTO CIENTÍFICO NA ESCOLA

\author{
Jhon Alexander Rico Quintero* / hidentidades@yahoo.com
}

Leonardo Tovar Bernal**/ leonardotovarbernal@yahoo.es

\section{Resumen}

El presente artículo describe la gestión, implementación y ejecución del Centro de Documentación en el Pensamiento Científico, CEDEPCI; una propuesta desarrollada en el Colegio Las Violetas I.E.D., de la localidad quinta (Usme) de Bogotá. Expone los aspectos teóricos e institucionales contemplados para fundamentar el proyecto y realiza una breve mención de las actividades pedagógicas realizadas por el CEDEPCI y del material que allí se encuentra. Propone, además, una reflexión sobre la importancia de estas iniciativas y las dificultades que enfrenta en la escuela.

\section{Summary}

This article describes the management, implementation and execution of CEDEPCI Centro de Documentación en el Pensamiento Científico-, a proposal developed at the Colegio Las Violetas I.E.D., of the town house (Usme) in Bogota. Exposes the theoretical and institutional aspects considered to support the project, and made a brief mention of the educational activities of the CEDEPCI and material found there. It also proposes a reflection on the importance of these initiatives and the difficulties faced in school.

\section{Resumo}

Este artigo descreve a gestão, implementação e execução do CEDEPCI - Centro de Documentación en el Pensamiento Científico-, uma proposta desenvolvida na Colegio Las Violetas I.E.D., da casa da cidade (Usme) em Bogotá. Expõe os aspectos teóricos e institucionais considerados para apoiar o projeto, e fez uma breve menção das atividades educacionais da CEDEPCI e material encontrado lá. Além disso, propõe uma reflexão sobre a importância dessas iniciativas e as dificuldades enfrentadas na escola.

\section{Palabras clave}

CEDEPCI, pensamiento científico, ciencia, escuela, estudiante.

\section{Keywords}

CEDEPCI, scientific thinking, science, school, student.

\section{Palavras chave}

CEDEPCI, pensamento científico, ciência, escola, estudante.

* Docente de Ciencias Sociales de Las Violetas I.E.D., Licenciado en Ciencias Sociales de la Universidad Distrital Francisco José de Caldas, candidato a Magister en Estudios Sociales de la Universidad Pedagógica Nacional.

** Docente de la Universidad Minuto de Dios (sede Madrid), docente de Ciencias Sociales de Las Violetas I.E.D., Licenciado en Ciencias Sociales de la Universidad Pedagógica Nacional, Magister en educación de la Universidad Pedagógica Nacional; correo electrónico: leonardotovarbernal@yahoo.es

Fecha de recepción: marzo 28 de 2014 / Fecha de aprobación: mayo 23 de 2014 


\section{Introducción}

El Centro de Documentación en el Pensamiento Científico, CEDEPCI, es un espacio destinado para despertar y fortalecer el conocimiento científico en la comunidad educativa del Colegio Las Violetas I.E.D. Se trata de una propuesta encaminada a crear una sala de documentación especializada en la que sea posible investigar y profundizar, desde una perspectiva científica, en las temáticas abordadas en las diferentes áreas del conocimiento que se imparten en la escuela. El proyecto surge por la necesidad de estimular el interés por la investigación y la ciencia. Con su implementación se pretendió generar un espacio de consulta, análisis y desarrollo de actividades dirigidas a fortalecer el pensamiento científico en los procesos académicos. Un lugar que propendiera por desarrollar el ejercicio investigativo como una herramienta didáctica y pedagógica.

\section{¿Por qué el CEDEPCI?}

El CEDEPCI se proyectó como una iniciativa que contribuyera con la realización del derecho a la educación, tal cual lo consagra el Artículo 68 de la Constitución, que señala que: "[...] es un derecho de la persona y un servicio público que tiene una función social; con ella se busca el acceso al conocimiento, a la ciencia, a la técnica, y a los demás bienes y valores de la cultura" (Constitución Política de Colombia, 1991). Además, la Ley 115 de 1994, Artículo 5, numerales 5 y 7, que regula su cumplimiento, procura alcanzar los siguientes fines: "La adquisición y generación de los conocimientos científicos y técnicos más avanzados [...] mediante la apropiación de hábitos intelectuales adecuados para el desarrollo del saber" (Ley 115, 1994).

Los estudiantes de Las Violetas deben enfrentar a diario condiciones socioeconómicas difíciles que les limitan el acercamiento a libros y materiales de consulta; situaciones que, vistas en conjunto, socaban las posibilidades de acceso a una educación de calidad. La realidad era, entonces, que la institución no contaba con un centro de documentación en el que alumnos y maestros pudieran ampliar y profundizar, a partir del estudio de materiales físicos, didácticos y virtuales, los temas que trabajan a diario. Así mismo, la falta de un espacio en el colegio en el que se potencializara el pensamiento científico, se constituyó en una de las mayores preocupaciones del grupo de docentes que impulsaron la propuesta.

Es cierto que la humanidad se encuentra en una época en la que el desarrollo científico ha dado pasos de gigante, tanto en la ciencia aplicada como en aquella que intenta explicar el mundo; pero más cierto aún es que los estudiantes de Las Violetas desconocen el origen de estos avances. A pesar de que hacen uso de los resultados de la ciencia aplicada a partir de la utilización de la Internet, el televisor, la radio, el celular o el computador, no entienden que éstos han sido el resultado del progreso científico se ha construido que por generaciones sobre la base de los fundamentos de la ciencia y del pensamiento científico.

Cuando el ser humano en su larga historia se acercó a la realidad y pretendió explicarla, lo hizo, grosso modo, de dos maneras. La primera, ha sido producto del "saber inmediato o espontaneo", obra del acercamiento involuntario de los sentidos (García, 1997, p. 27). Fue el fruto de la interacción cotidiana con las cosas y los hombres, caracterizada por el predominio de los sentidos y la carencia de reflexiones profundas y sistemáticas (García, 1997). Se trató, pues, de un conocimiento sustentado en las ideas generadas por el sentido común, que es impreciso y fragmentado, además de haber conseguido su extendida aceptación en la "autoridad no criticada de la costumbre" (Padilla, 1986, p.226).

Este tipo de conocimiento ha acompañado a la humanidad desde que inició su tránsito por la Tierra, jugando un papel de suma importancia para la sobrevivencia humana, convirtiéndose en no pocas ocasiones en el punto de partida para la investigación científica. Sin embargo, el pensamiento no puede detenerse allí. Si lo hiciera y la explicación humana se conformara con el saber generado por el sentido común y cotidiano, el conocimiento estaría confinado a la imprecisión y los razonamientos no irían "más allá del actual testimonio de nuestros sentidos o de los registros de la memoria" (Hume, 1789, citado en Padilla, 1986, p. 115). Se necesita, entonces, dar un paso adelante. 
La humanidad también ha recurrido al pensamiento científico para aproximarse y explicar el mundo y sus fenómenos. Esta segunda forma de conocimiento busca esclarecer y transformar la realidad basándose en la ciencia, entendida como un sistema de conocimientos racionales, sistemáticos, precisos, demostrables y falibles (Bunge, 1960), que han proporcionado a la humanidad las herramientas para hacer "inteligible el mundo" (Padilla, 1986, p. 225). Esta forma de abordar la realidad prescinde de las explicaciones sobrenaturales en todas sus formas, desde la magia y la superstición hasta los milagros y las hazañas de este o aquel libro sagrado. El pensamiento científico propende por los argumentos sólidos, va más allá de las apariencias, de las primeras impresiones, e indaga sobre las causas y los efectos que producen los fenómenos estudiados, tratando de "analizar sus diversas formas de desarrollo", así como "sus nexos internos" (Marx, 1959, p. XXIII). Las actividades desarrollas en el CEDEPCI proponen ir en consonancia con estos objetivos.

El CEDEPCI le apuesta a la formación del pensamiento científico en la escuela, y a que la ciencia se convierta en un "lenguaje para hablar del mundo" (Bronowsky, 1951, citado en Santilli, et al., 1998, p. 19). Son varias las razones que impulsan a ello. Es necesario que los alumnos se familiaricen con los conceptos y teorías producidos por la ciencia a lo largo de su historia para entender y transformar la realidad. Conocer los anales del pensamiento científico, con todas sus instituciones, su evolución y sus logros y dificultades (Santilli, et al., 1998) es ampliar la formación intelectual de los estudiantes y extender su mirada sobre cómo el ser humano ha pensado y construido buena parte de lo que él es hoy en día. Con los interrogantes o las desavenencias que puedan surgir, en la actualidad es posible observar que la ciencia y su aplicación "ha transformado la faz de la Tierra" y ha intervenido de manera decisiva en la construcción de la "civilización occidental" (Padilla, 1986, p. 224).

Sobre ello deben reflexionar los estudiantes y ser conscientes de cómo es que realmente se mueve el mundo. Aquí entra en juego el espíritu crítico que despierta el pensamiento científico, pues no se trata solo de aplicar lo que la ciencia nos provee, sino de aprender a pensar con los elementos que su filosofía nos proporciona. Así como ha servido para desmontar las concepciones equivocadas sobre el funcionamiento de la naturaleza, también será una excelente herramienta para preguntarse sobre el uso político o destructivo que se la ha dado, así como por las razones por las cuales se ha concentrado en pocas manos; Carl Sagan (1997, citado en Romanovich, 2010b), escribía al respecto:

"[...] la ciencia es más que un cuerpo de conocimiento, es una manera de pensar. Preveo cómo será la América de la época de mis hijos o nietos: [...] los temibles poderes tecnológicos estarán en manos de unos pocos y nadie que represente el interés público se podrá acercar siquiera a los asuntos importantes; la gente habrá perdido la capacidad de establecer sus prioridades o de cuestionar con conocimiento a los que ejercen la autoridad; nosotros, aferrados a nuestros cristales y consultando nerviosos nuestros horóscopos, con las facultades críticas en declive, incapaces de discernir entre lo que nos hace sentir bien y lo que es cierto, nos iremos deslizando, casi sin darnos cuenta, en la superstición y la oscuridad".

También es importante que los estudiantes comprendan que el desarrollo de la técnica y la ciencia han contribuido enormemente a la satisfacción de las necesidades humanas (Britto, 2004), y que los centros e instituciones más importantes del mundo, que lideran la investigación en todos los órdenes, se sustentan en la lógica del pensamiento científico: el MIT (Massachusetts Institute of Technology), la NASA (National Aeronautics and Space Administration ) o el CERN (European Organization for Nuclear Research). Es un hecho que:

"[...] las investigaciones serias realizadas en los diferentes campos del conocimiento lo siguen adoptando; las facultades de ciencia, los institutos científicos y la tecnología que se coloca en circulación o la que se reserva para intereses particulares, continúan sustentándose en el modelo propuesto por la ciencia moderna. Por lo demás, la mayoría de los seres humanos sigue utilizando el teléfono o la Internet para comunicarse; el televisor y la radio como una forma de ocio; la geografía y la astronomía para conocer la Tierra y el universo; el autobús, el tren o el avión para transportarse; la biología para entender el cuerpo humano o el de otras especies; el médico, los hospitales y la medicina para sanarse; y sigue recurriendo a la luz eléctrica para salir de la oscuridad" (Romanovich, 2010a). 


\section{Etapas de la propuesta}

El CEDEPCI se ha gestado en tres grandes momentos, los cuales han estado en permanente evaluación y han sido definidos según las actividades y procesos adelantados para su realización:

Fase de Gestión.

Fase de Implementación.

Fase de Ejecución.

\section{Fase de Gestión}

Se denomina Fase de Gestión al conjunto de procedimientos, actividades y demás acciones dirigidas a la aprobación del proyecto por parte de los órganos competentes del colegio. Este proceso se inició a finales del año 2011 tras la enunciación formal de la propuesta, junto con los requerimientos académicos, institucionales y legales solicitados para su aceptación.

Una vez reconocidas las consideraciones formales que justificaban la iniciativa, se presentó ante el Consejo Directivo del Colegio Las Violetas I.E.D., en el marco de lo que se denomina Proyectos de Inversión de Presupuesto Participativo, el cual es adjudicado a las instituciones educativas públicas. En este escenario se referenciaron las contribuciones académicas, pedagógicas, didácticas e investigativas que proporcionaría el CEDEPCI. El proyecto fue aprobado por plenaria y se procedió a la asignación presupuestal para su implementación en el año 2012. La adjudicación de presupuesto para el centro de documentación ha continuado para el 2013 y 2014.

La inversión para el desarrollo de la propuesta se aproxima a los cuatro millones de pesos y se espera que para el 2014 sea cercana a los tres millones. El presupuesto asignado ha estado dirigido a la compra de equipos e insumos y a la realización de actividades pedagógicas.

\section{Fase de Implementación}

La implementación corresponde a las acciones emprendidas para el funcionamiento del CEDEPCI, es decir, describe los modos de consecución de los recursos y material de diverso orden. Una vez se obtuvo la aprobación del proyecto, se dio inicio a su materialización, la cual implicó el empleo de diversas formas de gestión: inversión del presupuesto asignado, donación de material bibliográfico, equipos y otros insumos por parte de agentes internos y externos a la institución, y la recuperación de material subutilizado o en remate.

Dentro del proceso de implementación el proyecto ha contado con la garantía de poseer un espacio físico exclusivo en las instalaciones del colegio, el cual empezó a ser dotado de mobiliario, recursos eléctricos e informáticos, así como de material tecnológico, bibliográfico, audiovisual y didáctico. A la fecha, el CEDEPCI ha logrado conseguir los siguientes recursos, los cuales están disponibles para la ejecución de actividades afines al desarrollo científico de estudiantes y maestros:

Tabla 1. Lista de mobiliario, material y accesorios disponibles

\begin{tabular}{|c|c|c|c|}
\hline Cantidad & Descripción & Tipo & Observaciones \\
\hline 4 & Computadores portátiles & Tecnológico & $\begin{array}{c}\text { Con acceso a Internet, cada uno con sus } \\
\text { respectivos parlantes, audífonos y mouse. }\end{array}$ \\
\hline 1 & Televisor plasma & Tecnológico & $\begin{array}{c}\text { Con cables HDMI, VGA, RCA y USB } \\
\text { para conectar a los computadores } \\
\text { y al Teatro en Casa. }\end{array}$ \\
\hline 1 & Teatro en Casa & Tecnológico & \\
\hline 1 & Impresora - Escáner & Tecnológico & \\
\hline 1 & Reproductor de VHS & Tecnológico & \\
\hline 1 & Rebobinador de VHS & Tecnológico & \\
\hline 24 & Mesas de estudio & Mobiliario & \\
\hline 40 & Sillas & Mobiliario & \\
\hline
\end{tabular}




\begin{tabular}{|c|c|c|c|}
\hline 3 & Archivadores & Mobiliario & \\
\hline 1 & Revistero & Mobiliario & \\
\hline 5 & Armarios & Mobiliario & $\begin{array}{l}\text { Disponible para almacenar material } \\
\text { bibliográfico y audiovisual. }\end{array}$ \\
\hline 4 & Muebles para computador & Mobiliario & \\
\hline 110 & Títulos bibliográficos & Bibliográfico & $\begin{array}{l}\text { Material en diferentes áreas del } \\
\text { conocimiento científico, diccionarios, } \\
\text { revistas y enciclopedias. }\end{array}$ \\
\hline 150 & Títulos de películas & Audiovisual & $\begin{array}{l}\text { Material afín a temas científicos: } \\
\text { películas, series y documentales. }\end{array}$ \\
\hline 25 & Láminas cartográficas & Didáctico & Láminas geográficas e históricas \\
\hline 1 & Microscopio didáctico & Didáctico & \\
\hline 1 & Globo Terráqueo & Didáctico & \\
\hline
\end{tabular}

La sala asignada en un principio fue reubicada, luego de que el primer espacio presentara dificultades relacionadas con la acústica, la luminosidad y el tamaño. La necesidad de cambiar de aula fue producto de la evaluación generada tras el primer año de implementación del CEDEPCI, en el 2012.

El CEDEPCI pretende seguir con la tarea de ampliar el material disponible, por ejemplo, para el 2014 se contará con el presupuesto necesario para la afiliación a una revista científica. Espera, igualmente, contar con la asignación de presupuesto proveniente de los proyectos participativos y continuar con la gestión de recursos con entidades privadas y oficiales. El material adquirido se encuentra disponible para la comunidad educativa y es administrado por los docentes responsables del proyecto en colaboración permanente con los estudiantes. A propósito, cabe destacar y agradecer profundamente a los alumnos de grado noveno y décimo que colaboraron con el CEDEPCl, en especial, y por su dedicación y compromiso, a las estudiantes Dina Luz Cortés y Carolina Marín, quienes, con su trabajo, ayudaron significativamente en el desarrollo del centro de documentación.

\section{Fase de Ejecución}

Comprende las acciones orientadas al funcionamiento y sostenibilidad de la sala y a los trabajos pedagógicos e investigativos que promuevan el pensamiento científico en la comunidad educativa. Se distinguen así dos momentos, que permiten dar cuenta de la ejecución del proyecto:
Funcionamiento de la sala.

Actividades pedagógicas e investigativas.

\section{Funcionamiento de la sala}

El Centro de Documentación en el Pensamiento Científico, entendido como una sala que alberga material relacionado con diferentes campos de la ciencia, ha diseñado una serie de instrumentos y de estrategias para su funcionamiento, que garantizan unas dinámicas organizativas y proporcionan un permanente examen y actualización, lo cual ha redundado positivamente en su sostenimiento y desarrollo. A continuación, se describirán algunos de ellos:

\section{Folleto y correo oficial}

Se diseñó un folleto informativo con las características generales del proyecto, que sirvió como instrumento de difusión al interior de la comunidad educativa. Del mismo modo, se habilitó el correo institucional de la sala: cedepcilasvioletasied@gmail.com

\section{Ficha de solicitud de la sala T5}

Es un formato que será diligenciado por el estudiante, docente o administrativo docente responsable de cualquier actividad en el CEDEPCI. Aparte de ser un formato en donde se relaciona el material solicitado, allí se describe el objetivo pedagógico de la actividad y su relación con el estímulo y fortalecimiento del espíritu científico e investigativo: 
Tabla 2. Ficha de solicitud CEDEPCI

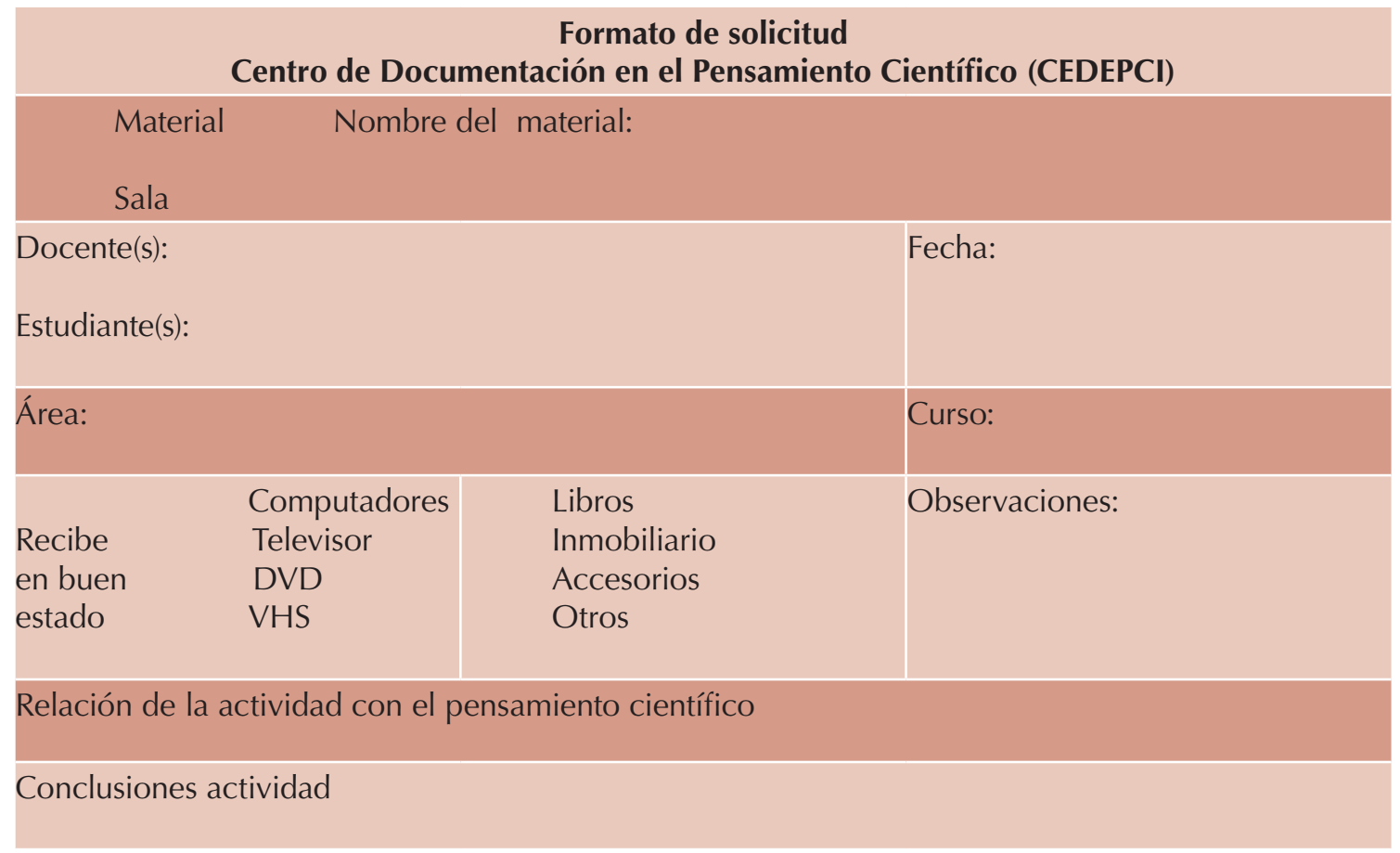

\section{Fichas material audiovisual}

Con el objetivo de caracterizar el material audiovisual, se implementó un modelo de ficha técnica ${ }^{1}$ en el que se consiga la información básica y los aportes pedagógicos que de éste se pueden obtener:

Tabla 3. Ficha Técnica utilizada para caracterizar material audiovisual

\begin{tabular}{|c|c|}
\hline \multicolumn{2}{|r|}{ Ficha Técnica No 1} \\
\hline Título & Cosmos: Un viaje personal1 \\
\hline Género & Documental \\
\hline Dirección & Adrian Malone \\
\hline Reparto & Carl Sagan (Presentador) \\
\hline País & Estados Unidos \\
\hline Año & 1980 \\
\hline Duración & 60 minutos cada episodio \\
\hline Sinopsis & $\begin{array}{l}\text { "Serie documental de divulgación científica escrita por Carl Sagan, Ann Druyan y Steven Soter } \\
\text { (con Sagan como guionista principal) [...] El programa de televisión estuvo listo en } 1980 \text { y constó } \\
\text { de trece episodios, cada uno de aproximadamente una hora de duración. La música utilizada } \\
\text { fue mayormente obra de Vangelis, y otros. Ganó un Premio Emmy y un Peabody. La serie se } \\
\text { ha emitido en } 60 \text { países y ha sido vista por más de } 500 \text { millones de personas. Tras el rodaje } \\
\text { de la serie, Sagan escribió el libro homónimo Cosmos, complementario al documental". }\end{array}$ \\
\hline Interés Didáctico & $\begin{array}{l}\text { Difusión de la "historia de la astronomía y de la ciencia [y del] del origen de la } \\
\text { vida. Concienciar sobre el lugar que ocupa nuestra especie y nuestro planeta en } \\
\text { el universo [dar a] conocer las modernas visiones de la cosmología y las últimas } \\
\text { noticias de la exploración espacial; en particular, las misiones Voyager". }\end{array}$ \\
\hline
\end{tabular}

1 El modelo de ficha técnica fue obtenido desde: http://www.buenosaires.gob.ar/areas/educacion/recursos/ed-ambiental/fichas-peliculas-ficcion.pdf 


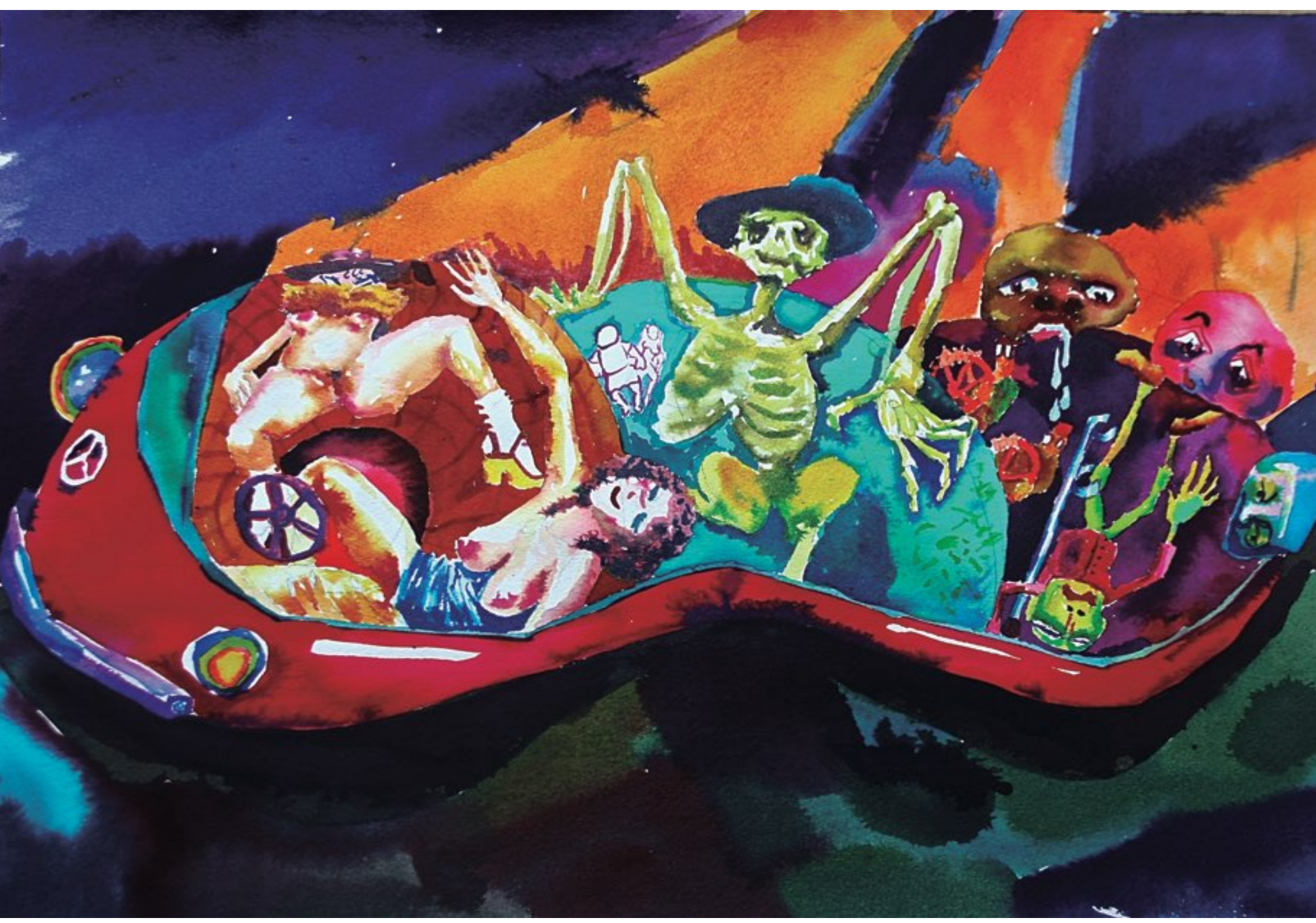

Luis Antonio Sánchez León 》 Título: Hora Feliz ॥ Técnica: Acuarela ॥ Tamaño: 27.7 X 41 Cm ॥ Año: 2013 


\section{Base de datos}

Con el objetivo de administrar la información, y para que la comunidad educativa pueda acceder a ella, se desarrolló una base de datos que facilita la sistematización del material existente en la sala. A continuación, se puede observar una imagen del software diseñado para este fin:

\section{Actividades pedagógicas e investigativas}

Junto la obtención de productos relacionados con la adecuación física del centro de documentación, se han realizado actividades de orden pedagógico e investigativo orientadas al estímulo del pensamiento científico y al reconocimiento del CEDEPCI como un centro de desarrollo académico:

\section{Logotipo del CEDEPCI}

Mediante la convocatoria a un concurso de dibujo, los estudiantes de los grados sexto y séptimo participaron en el diseño del logotipo del CEDEPCI. En este evento los niños y jóvenes representaron artísticamente imágenes alusivas a la ciencia y a los objetivos del proyecto. Para el diseño y construcción del modelo final se tuvieron en cuenta los aportes de las mejores propuestas. Esta actividad, así como la elaboración final del logotipo, estuvo a cargo del docente del área de artes Alexander Pérez.

\section{Teorías científicas}

La identificación y el estudio de algunas de las teorías científicas más importantes 2 fue una de las actividades pedagógicas impulsadas desde el centro de documentación. Se trató de un ejercicio dirigido a los estudiantes del grado décimo de las dos jornadas del colegio. Allí, los jóvenes emprendieron su estudio a partir de unas lecturas preliminares, que complementaron con material audiovisual e investigación extra escolar que daban cuenta del nombre, autor, época, planteamientos generales e importancia de cada una de ellas. A cada grupo se le asignó una teoría para que la profundizara, la expusiera y la representara por medio de un modelo a escala. Fue un trabajo que contó con la colaboración permanente

2 Modelo Heliocéntrico, Ley de Gravitación Universal, Átomo (diferentes modelo del átomo), Evolución de las especies y del hombre, Herencia Genética, Relatividad, Física Cuántica, Deriva Continental, Origen de la vida, Big Bang, Estructura del ADN, Proyecto Genoma Humano, Bosón de Higgs. del área de artes, que asesoró a los grupos en cuanto a los materiales más apropiados para dar a entender el concepto que los alumnos deseaban expresar.

Así mismo, en el marco de esta actividad, fue posible la visita al museo de Historia Natural, de la Universidad Nacional de Colombia, y al Museo Geológico y Paleontológico José Royo y Gómez, de INGEOMINAS. Esta salida pedagógica contribuyó significativamente en el desarrollo del pensamiento científico, y sirvió para que los estudiantes establecieran relaciones entre lo trabajado en la sala de documentación y las evidencias que estos museos pueden aportar para la confirmación de algunas de las teorías, así como para el reconocimiento del estrecho vínculo entre ellas y las disciplinas científicas (la Evolución y la Deriva Continental, con la geología).

Las actividades mencionadas marcan el inicio de un conjunto de propuestas académicas dirigidas a la consolidación de los objetivos trazados por el CEDEPCI. Se espera seguir ampliando la cantidad de eventos centrados en el desarrollo del pensamiento científico, no solo para estudiantes, sino para la comunidad educativa en general.

\section{Relación con otros proyectos}

El CEDEPCI ha estrechado su relación con otras áreas y proyectos de la institución. Entre las experiencias desarrolladas se destaca la realización de una exposición fotográfica referente a la afrocolombianidad; una actividad a la que asistió gran parte de la comunidad educativa y que fue llevada a cabo en mayo del 2012. Su objetivo se centró en destacar el desempeño de personas de ascendencia afro a nivel mundial en el deporte, las artes, la política y la ciencia. El evento fue organizado desde el CEDEPCI y se realizó en sus instalaciones (en el primer espacio físico asignado).

Por otra parte, el centro de documentación se ha aprovechado como espacio para el concurso de ortografía y cuentos infantiles, también allí se han desarrollado actividades académicas relacionadas con ciencias sociales, biología, química, matemáticas, música, artes y filosofía. Sirvió también como escenario de la exposición sobre las culturas prehispánicas; actividad que contó con el material de maletas didácticas facilitadas por el Museo del Oro, las cuales fueron gestionadas 
por el área de artes. Igualmente, el CEDEPCI ha facilitado sus instalaciones y equipos para el acercamiento a las nociones de la astronomía, mediante el empleo de herramientas como el Stellarium; un proceso adelantado por el docente de matemáticas de la jornada tarde, Oscar Caballero.

El proyecto ha intentado responder a los objetivos institucionales a través de su incorporación a las iniciativas eco-pedagógicas y a los proyectos de ruralidad que definen al colegio. Para el futuro, con el ánimo de fortalecer los procesos académicos y de promover nuevas formas de financiación, se buscará la vinculación con programas como ONDAS, que dirige COLCIENCIAS en asocio con la Universidad Pedagógica Nacional y la Alcaldía Distrital.

Finalmente, basta mencionar que cada uno de los momentos que constituyen el proyecto se encuentra sujeto a la evaluación interna y externa, posibilitando la realización de los ajustes correspondientes. Esto ha contribuido, por ejemplo, a que las condiciones logísticas y físicas mejoren, el material y las actividades pedagógicas aumenten, se visualice institucionalmente la propuesta y el número de participantes sea cada vez mayor.

\section{Conclusiones}

El CEDEPCI se convierte en una experiencia que posibilita la relación entre disciplinas e incentiva el pensamiento científico en los jóvenes. Se ha observado cómo la ciencia y el pensamiento que de ella se genera van más allá de las valiosas contribuciones de la técnica aplicada o la explicación esotérica ${ }^{3}$ de un fenómeno (Kuhn, 1971), el pensamiento científico contribuye notablemente al esclarecimiento del pensamiento general y a que las personas se construyan una opinión informada, justa y ajustada con la realidad (Cordón, 1976). En una palabra, despierta, y provee de argumentos sólidos, de espíritu crítico.

3 Entendida como explicaciones que en un principio, debido al lenguaje técnico y poco reconocido por los no especialistas, carecen de difusión, lo que las hace cerradas e inasequibles. Es lo que, entre otras cosas, Kuhn (1971) denomina "investigación más profesional y esotérica".
Entre las fortalezas que presenta la implementación del proyecto, se destacan la colaboración entre los diferentes estamentos de la institución en la materialización de los objetivos. Se evidencia así una relación institucional que involucra al sector administrativo, que provee los recursos; los docentes, que observan el centro de documentación como un escenario alternativo para ahondar en sus propósitos educativos; los estudiantes, que se sienten identificados con el ejercicio científico e investigativo; y los padres de familia, que reconocen en él, tanto un espacio para la consulta y el desarrollo de actividades que requieren sus hijos, como una alternativa a las carencias materiales y culturales propias del sector socio-económico en el que viven.

Además, vincula la comunidad educativa a los procesos institucionales y contribuye a la interacción del ejercicio de aula con otros escenarios de aprendizaje. Se constituye en un escenario que permite evidenciar diferentes elementos epistémicos, éticos, pedagógicos y didácticos que actúan en función del desarrollo de iniciativas orientadas al trabajo teórico y práctico con los estudiantes.

Este tipo de propuestas está sujeto a varias dificultades, las relacionadas con la imposibilidad de sostenerlas en el tiempo son las más apremiantes; por un lado, si la comunidad no se apropia de ellas, no sobrevivirán más allá del esfuerzo que puedan realizar quienes las propusieron en principio, pues cuando éstos no estén es posible que no haya quien los asuma como propios y la iniciativa termine; por el otro, la dependencia financiera y administrativa mina su estabilidad, en tanto que cuando no se disponga de recursos suficientes o una administración no desee continuar con el proyecto, éste desaparecerá.

Las iniciativas pedagógicas impulsadas desde la escuela presentan las mismas dificultades que las de la educación en general, en tanto que su efectividad y posibilidades de incidencia se encuentran sujetas a los límites impuestos por el colegio, es decir, ¿puede la escuela responder por lo que sucede por fuera de ella?; ¿tiene la capacidad de hacerlo?; ¿es su responsabilidad? Cualquier iniciativa fomentada desde la educación debe considerar su verdadera capacidad de transformación, pues se encuentra condicionada por 
factores externos que la determinan, tanto a ella misma, como a la población a la cual está dirigida; tan solo desde la educación es muy difícil cambiar estructuras de pensamiento arraigadas durante años.

La escuela no es la única que incide en la vida de los estudiantes, por el contrario, otras instancias como la familia, la religión, el barrio, los medios masivos de comunicación o las redes sociales parecen disfrutar de un influjo mayor en la formación de los jóvenes. De modo que la efectividad de procesos pedagógicos como el CEDEPCI debe enfrentar dificultades de este orden, ya que el contacto que los estudiantes pudieran tener con ellos, en número de horas y en profundidad, es inferior al logrado con las instancias mencionadas.

Es en esta perspectiva que se deben entender, no solo los procesos pedagógicos adelantados de manera individual o colegiada, sino toda iniciativa que se promueva desde la educación. La educación es un valor imprescindible para las naciones, y lo que desde allí se promueva de seguro tendrá una preponderancia notable; sin embargo, ella no es la médula de la sociedad y ésta no gira exclusivamente a su alrededor; menos aún, no es la única institución que genera y soluciona problemas. No tiene porque llevar, entonces, todo el peso de las dificultades sociales.

\section{Referencias}

Britto, L. (2004). La ciencia: fundamentos y método. Caracas: Consejo Nacional de la Cultura (Biblioteca Básica Temática).

Bunge, M. (1960). La ciencia, su método y su filosofía. Obtenido el 19 de Diciembre de 2013, desde: http://iner.udea.edu. co/especializacion6/epistemologia/Mario_Bunge_La\%20ciencia_su_m\%E9todo_y_su_filosof\%EDa.pdf

Cordón, F. (1976). Pensamiento general y pensamiento científico. Madrid: Ayuso.

Fichas técnicas películas de ficción. Obtenido el 7 de Mayo de 2013, desde: http://www.buenosaires.gob.ar/areas/educacion/ recursos/ed-ambiental/fichas-peliculas-ficcion.pdf

García Duque, C. E. (1997). Evolución histórica del pensamiento científico: desde la antigüedad clásica hasta el período moderno. Manizales: Universidad de Manizales.

Kuhn, T. (1971). La estructura de las revoluciones científicas. Bogotá: Fondo de Cultura Económica.

Ministerio de Educación. (1994). Ley 115 de 1994. Recuperado el 1 de Noviembre de 2011, desde: http://www.alcaldiabogota. gov.co/sisjur/normas/Norma1.jsp?i=292

Marx, C. (1959). El capital. Critica de la economía política (Vol.1). México: Fondo de Cultura Económica.

Padilla, H. (1986). El pensamiento científico. México: Editorial Trillas.

República de Colombia. (1991). Constitución Política de Colombia. Obtenido el 1 de Noviembre de 2011, desde: http://www. alcaldiabogota.gov.co/sisjur/normas/Norma1.jsp?i=4125

Romanovich, L. N. (2010a). Sobre la postmodernidad. Obtenido el 19 de Diciembre de 2013, desde: http://www.notasobreras. net/index.php/nacional/politica/70-ciencia/268-sobre-la-postmodernidad

Romanovich, L. N. (2010b). El conocimiento en la "sociedad del conocimiento". Obtenido el 19 de Diciembre de 2013, desde: http://www.notasobreras.net/index.php/ciencia/317-elconocimiento-en-la-sociedad-del-conocimiento

Sagan, C. (1980). Cosmos: Un viaje personal. Obtenido el 7 de Mayo de 2013, desde: http://es.wikipedia.org/wiki/Cosmos:_un_viaje_personal

Santilli, E., et al. (1998). El pensamiento científico. Buenos Aires: Editorial Biblos.

\section{(Footnotes)}

1 La información de la ficha técnica fue tomada de: http:// es.wikipedia.org/wiki/Cosmos:_un_viaje_personal 


\section{Diálogo del conocimiento}

El texto escrito por los profesores Jhon Alexander Rico Quintero, Leonardo Tovar Bernal, describe la gestión, implementación y ejecución del Centro de Documentación en el Pensamiento Científico, CEDEPCI; una propuesta desarrollada en el Colegio Las Violetas I.E.D., de la localidad quinta (Usme) de Bogotá. Este artículo nos muestra el trabajo desarrollado por los maestros en esta institución, que enriquece a los alumnos desde todas las aristas que se mire, dando paso a que en cualquier circunstancia o lugar de Colombia puedan llevar adelante propuestas que cambien la vida de nuestros estudiantes en América Latina y de las instituciones a las cuales pertenecemos.

Los pasos que se muestran en este trabajo guían de manera clara el proceso para llevar a cabo un proyecto en nuestros planteles, empezando por cuál o cuáles son nuestras necesidades y desde allí se genera la pregunta: qué necesitamos hacer o qué hacemos para cambiar nuestro entorno, si tenemos este cuestionamiento como base para avizorar lo que podemos hacer ya hemos avanzado en la mitad del camino, como se demuestra en este escrito.

El artículo muestra de manera clara el proceso con todos sus bemoles, nada es fácil y menos en su hermosa Colombia, pero al final se logra como lo hacemos los maestros latinoamericanos sacar adelante lo que nos ponemos como batalla en el aula y de esta manera cambiar la vida de nuestros estudiantes y del lugar en el que habitamos, muchas gracias a nuestros maestros por hacer posible estos sueños.

Alcibíades Brazan 